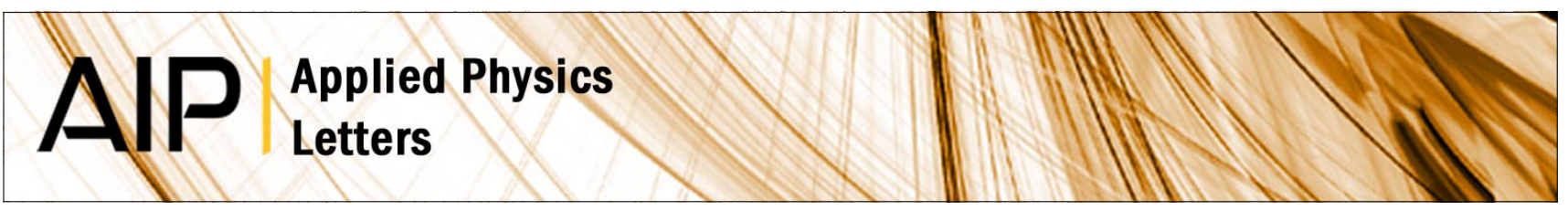

\title{
Coherent anti-Stokes Raman microspectroscopy using spectral focusing with glass dispersion
}

Israel Rocha-Mendoza, Wolfgang Langbein, and Paola Borri

Citation: Appl. Phys. Lett. 93, 201103 (2008); doi: 10.1063/1.3028346

View online: http://dx.doi.org/10.1063/1.3028346

View Table of Contents: http://apl.aip.org/resource/1/APPLAB/v93/i20

Published by the American Institute of Physics.

\section{Related Articles}

Low-damping spin-wave propagation in a micro-structured Co2Mn0.6Fe0.4Si Heusler waveguide Appl. Phys. Lett. 100, 112402 (2012)

Long-range orientation correlation in liquids

J. Chem. Phys. 136, 044503 (2012)

Isothermal crystallization of poly(3-hydroxybutyrate) studied by terahertz two-dimensional correlation spectroscopy

Appl. Phys. Lett. 100, 011907 (2012)

Eliminating the broadening by finite aperture in Brillouin spectroscopy

Rev. Sci. Instrum. 82, 113110 (2011)

Effects of Sr content and bias field on acoustic properties of strontium barium niobate studied by Brillouin light scattering

Appl. Phys. Lett. 99, 212902 (2011)

\section{Additional information on Appl. Phys. Lett.}

Journal Homepage: http://apl.aip.org/

Journal Information: http://apl.aip.org/about/about_the_journal

Top downloads: http://apl.aip.org/features/most_downloaded

Information for Authors: http://apl.aip.org/authors

\section{ADVERTISEMENT}

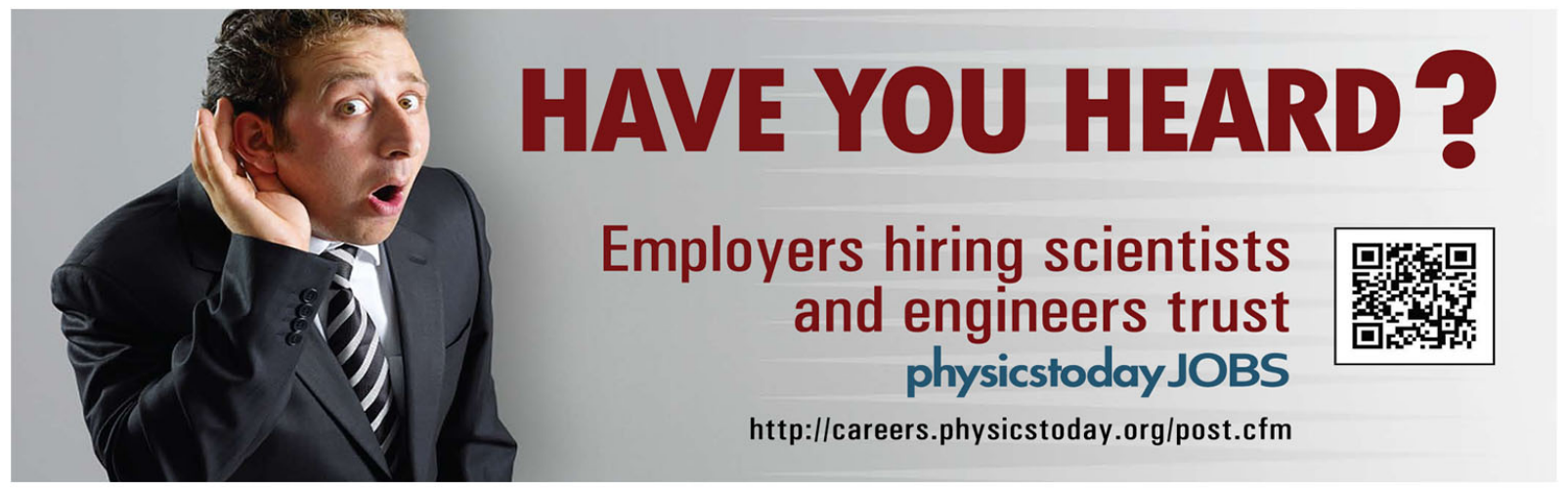




\title{
Coherent anti-Stokes Raman microspectroscopy using spectral focusing with glass dispersion
}

\author{
Israel Rocha-Mendoza, Wolfgang Langbein, and Paola Borri ${ }^{\mathrm{a})}$ \\ School of Biosciences and School of Physics and Astronomy, Cardiff University, Museum Avenue, \\ Cardiff CF10 3US, United Kingdom
}

(Received 8 September 2008; accepted 29 October 2008; published online 18 November 2008)

\begin{abstract}
We demonstrate experimentally that coherent anti-Stokes Raman microspectroscopy with high spectral resolution is achieved using femtosecond laser pulses chirped up to a few picoseconds by glass elements of known group-velocity dispersion without significant intensity losses. By simply choosing the length of the glass, the chirp of Stokes and pump pulses is tailored to obtain a spectral resolution given by the Fourier limit of the chirped pulse duration. We show that for chirped pulse durations shorter than or comparable to the Raman coherence time, maximum signal occurs for a pump arriving after the Stokes pulse, a time-ordering effect confirmed by numerical simulations. (C) 2008 American Institute of Physics. [DOI: 10.1063/1.3028346]
\end{abstract}

Coherent anti-Stokes Raman scattering (CARS) microscopy has recently emerged as a noninvasive technique for imaging in cell biology, offering three dimensional sectioning capability and chemical specificity without the need of staining and fluorescence tagging. ${ }^{1}$ A variety of CARS schemes have been proposed in order to improve the sensitivity and selectivity of this technique. ${ }^{2-4}$ Since typical Raman resonances have coherence times in the picosecond range, their coherent excitation is optimized in spectral selectivity using picosecond pulses. However, tunable picosecond sources are less available and are also less efficient for two-photon fluorescence (TPF) or second-harmonic generation (SHG), well known imaging modalities which lend themselves to be implemented together with CARS in the same microscope.

For a two-pulse CARS with pump and Stokes field $E_{P}$ and $E_{S}$ the vibrational excitation is governed by the interference term $E_{P} E_{S}^{*}$. The spectral resolution is thus not determined by the spectrum of the individual exciting pulses but by the spectrum of their temporal interference. Consequently, it is possible to use femtosecond pulses and still achieve high spectral resolution by controlling their spectral phase without loss of pulse energy. Furthermore, achieving the spectral selectivity by the excitation allows the detection of CARS by fast and efficient single-channel detectors similar to those used for confocal fluorescence, TPF, or SHG microscopy. This is in contrast to techniques using femtosecond pulses for broadband excitation and spectrally resolved detection. 5,6

A straightforward way to exploit phase shaping in order to achieve high spectral resolution in CARS was demonstrated by Hellerer et $a .^{7}$ and termed "spectral focusing." In this scheme, an equal linear temporal chirp of both pump and Stokes pulses creates a constant instantaneous frequency difference (IFD) between the pulses. The resulting spectral width of $E_{P} E_{S}^{*}$ centered at the IFD is given by the Fourier limit of the temporal envelope of the pulses, which can be elongated by the applied linear chirp to many picoseconds. This approach additionally allows to tune the IFD within the spectral width of the pulses by simply adjusting their relative time delay, allowing to perform CARS spectroscopy without tuning the laser sources. A more complex approach was re-

${ }^{\text {a)} E l e c t r o n i c ~ m a i l: ~ b o r r i p @ c a r d i f f . a c . u k . ~}$ ported by Dudovich et al. ${ }^{8}$ and employs a sinusoidal phase shaping creating a pulse train but achieves a spectral resolution only after measuring with pulse trains of different periods.

In this work we demonstrate that a constant IFD needed for spectral focusing is obtained by using the dispersion in high-index glass elements, significantly simplifying the pulse shaper design as compared to spatial light modulators or grating-lens based systems used in Refs. 7 and 8. We investigate the resulting spectral resolution as a function of the chirped pulse duration and characterize the influence of the temporal overlap of the pulses on the signal intensity.

The optical setup is sketched in Fig. 1. The Stokes beam

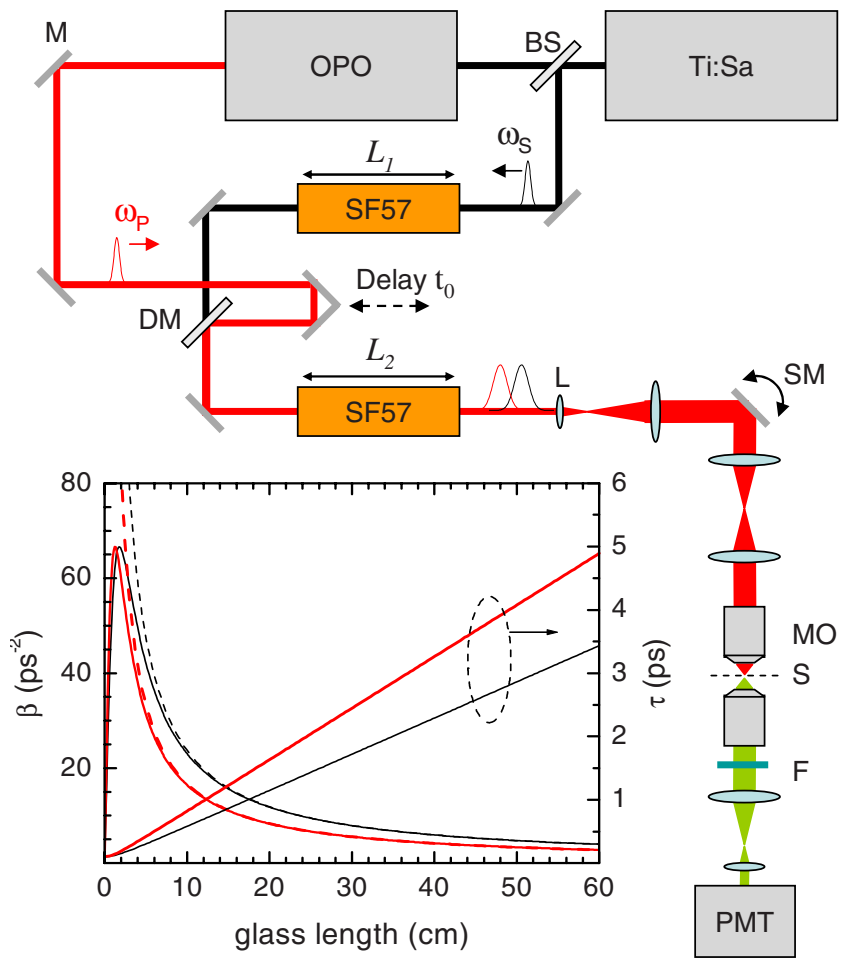

FIG. 1. (Color online) Sketch of the experimental setup. BS: beam splitter; M: mirror; SF57: glass blocks; DM: dichroic mirror; L: lens; SM: scanning mirror; MO: objective; S: sample; F: filter; PMT: photomultiplier tube. Graph: calculated $\beta(z)$ parameter and $\tau(z)$ for the pump (bold) and Stokes (thin) beams. Dashed traces are approximations for $\beta(z)$ (see text). 
is generated by a Ti:sapphire laser system which delivers transform-limited $100 \mathrm{fs}$ pulses centered at $832.5 \mathrm{~nm}$ at $76 \mathrm{MHz}$ repetition rate. These pulses are also used to synchronously pump an optical parametric oscillator, the signal of which is intracavity frequency doubled to provide the CARS pump pulses. Pump pulses ranging from 700 to $650 \mathrm{~nm}$ can be used to excite vibrational resonances from 2300 to $3400 \mathrm{~cm}^{-1}$. The Stokes pulses travel through a length $L_{1}$ of glass (SF57) and are collinearly overlapped with the pump pulses by a dichroic mirror. Both pulses then travel through a length $L_{2}$ of SF57 and are coupled into a homebuilt microscope. For the data shown in this article the average power of both pump and Stokes at the rear aperture of the input objective was $\sim 3 \mathrm{~mW}$. The temporal overlap between pump and Stokes pulses in the sample is adjusted via a delay stage and is specified by the delay time $t_{0}$, positive for pump leading. A pair of microscope objectives (Leica, Water immersion, numerical aperture of 1.2) is used to focus the exciting beams and collect the CARS signal. Spatially resolved images are obtained by beam scanning and/or moving the sample with a motorized/piezoelectric stage. The forward CARS signal is selected by a bandpass filter at $562 \pm 20 \mathrm{~nm}$ and detected by a photomultiplier.

An initially unchirped (transform-limited) Gaussian pulse with an electric field $E \propto \exp \left(-t^{2} / \tau_{\mathrm{G} 0}^{2}+i \omega_{0} t\right)$ of center frequency $\omega_{0}$ and duration [intensity full width at half maximum $(\mathrm{FWHM})] \tau_{0}=\tau_{\mathrm{G} 0} \sqrt{2 \ln 2}$ propagating through a length $z$ of dispersive material acquires ${ }^{9}$ an instantaneous frequency $\omega(t)=\omega_{0}+2 \beta t$ and a duration

$$
\tau(z)=\tau_{0} \sqrt{1+\left(\frac{4 \ln 2\left|k^{\prime \prime}\right| z}{\tau_{0}^{2}}\right)^{2}},
$$

with the linear chirp parameter $\beta(z)=8(\ln 2)^{2}\left|k^{\prime \prime}\right| z \tau_{0}^{-2} \tau^{-2}$ and the group-velocity dispersion (GVD) parameter of the material,

$$
k^{\prime \prime}=\frac{\lambda^{3}}{2 \pi c^{2}} \frac{d^{2} n}{d \lambda^{2}},
$$

where $c$ is the speed of light and $n$ is the index of refraction of the material at wavelength $\lambda$. The refractive index of glass is well described by the Sellmeier equation $n^{2}(\lambda)=1+\sum_{m=1}^{3} A_{m} \lambda^{2} /\left(\lambda^{2}-B_{m}\right)$. For the highly dispersive Schott glass SF57, the coefficients $\operatorname{are}^{10}\left(A_{1}, A_{2}, A_{3}\right)=(1.8165,0.4289,1.072)$ and $\left(B_{1}, B_{2}, B_{3}\right)$ $=(0.01437,0.05928,121.42) \mu \mathrm{m}^{2}$.

The resulting $\beta(z)$ and $\tau(z)$ are given in Fig. 1 for the center wavelength of Stokes $(832.5 \mathrm{~nm})$ and pump $(662.5 \mathrm{~nm})$ pulses of $\tau_{0}=102 \mathrm{fs}$ (determined by the pulse spectral width of $144 \mathrm{~cm}^{-1}$ FWHM). For large glass lengths $z \gg \tau_{0}^{2} /\left|k^{\prime \prime}\right|$, for which $\tau \gg \tau_{0}$, the chirp parameter can be approximated to $\beta^{-1} \approx 2 z\left|k^{\prime \prime}\right|$, independent of the initial pulse width $\tau_{0}$. In this case, the same linear chirp for Stokes and pump $\left(\beta_{S}=\beta_{P}\right)$ is achieved just by using a fixed ratio between the glass length of Stokes and pump, $L_{S} / L_{P}=k_{P}^{\prime \prime} / k_{S}^{\prime \prime}$ $\approx 1.42$ with $k_{P}^{\prime \prime}=k^{\prime \prime}(662.5 \mathrm{~nm})$ and $k_{S}^{\prime \prime}=k^{\prime \prime}(832.5 \mathrm{~nm})$. For our setup, these lengths are given by $L_{S}=L_{1}+L_{2}+L_{\mathrm{MO}}$ and $L_{P}$ $=L_{P 0}+L_{2}+L_{\mathrm{MO}}$, with the effective SF57 length of the microscope objectives $L_{\mathrm{MO}} \approx 2 \mathrm{~cm}$ (deduced from the measured GVD in the objective) and the initial chirp of the pump described by $L_{P 0} \approx 5 \mathrm{~cm}$ (deduced from the measured secondorder autocorrelation). Nonlinear chirp introduced by higher order material dispersion results for the present pulse parameters in a variation of the IFD of less than $0.1 \mathrm{~cm}^{-1}$ over the pulse duration $\tau$, negligible compared to typical Raman linewidths. Furthermore, we measured an extinction coefficient for the SF57 of less than $0.8 \mathrm{~m}^{-1}$, allowing for more than $70 \%$ transmission even for the biggest glass lengths used in this work while pulse shaper as in Refs. 7 and 8 has typically less than $50 \%$ transmission. Using glass to achieve spectral focusing is thus efficient, reproducible, and cost effective.

The spectral selectivity of the chirped excitation is determined by the intensity modulation driving the vibrations

$E_{P} E_{S}^{*} \propto \exp \left[-t_{0}^{2} / 2 \tau_{G}^{2}-2\left(t+t_{0} / 2\right)^{2} / \tau_{G}^{2}+i\left(\omega_{P}-\omega_{S}+2 \beta t_{0}\right) t\right]$,

having a power spectrum of $\exp \left[-\left(\omega-\omega_{D}\right)^{2} \tau_{G}^{2} / 4-t_{0}^{2} / 2 \tau_{G}^{2}\right]$ centered at the IFD $\omega_{D}\left(t_{0}\right)=\omega_{P}-\omega_{S}+2 \beta t_{0}$ with a FWHM of $\sigma_{D}=4 \sqrt{\ln 2} / \tau_{G}$. These expressions show that a high spectral resolution can be achieved with chirped pulses (numerically $\sigma_{D}=20.8 \mathrm{~cm}^{-1}$ for $\tau=1 \mathrm{ps}, \tau=\tau_{G} \sqrt{2 \ln 2}$ ) and that the center frequency of the modulation can be tuned by changing the delay $t_{0}$. However, besides providing spectral tunability, $t_{0}$ influences also the readout of the vibration by the pump. A vibrational resonance at $\omega_{r}$ responds to the excitation with a finite coherence time $T_{2}$. Therefore, the time ordering between creation of the vibration and its readout by the pump matters, giving maximum CARS for readout after creation $\left(t_{0}<0\right)$.

To demonstrate experimentally the spectral selectivity of chirped pulse and the effect of time ordering, we have investigated the CARS signal from the aromatic $\mathrm{C}-\mathrm{H}$ vibration at $\omega_{r}=3054 \mathrm{~cm}^{-1}$ in polystyrene beads of $3 \mu \mathrm{m}$ diameter, immersed in a gel of $2 \%$ agarose and $98 \%$ water (angular frequencies are implicitly converted to wave numbers dividing by $2 \pi c$ ). A CARS image of a cross section through the center of a bead is shown in the left inset of Fig. 2(b), from which we infer a lateral spatial resolution of $\sim 0.3 \mu \mathrm{m}$. CARS spectra were obtained from line scans across the bead as a function of $t_{0}$. The example given in the right inset of Fig. 2(b) illustrates how in one of these scans we could measure both the nonresonant CARS from the gel $\left(I_{\mathrm{nr}}\right.$ at $\left.x_{\mathrm{nr}}\right)$ and the resonant CARS from polystyrene $\left(I_{\mathrm{r}}\right.$ at $\left.x_{\mathrm{r}}\right)$. The resulting CARS spectra for three different chirped pulse durations $\tau$ are shown in Fig. 2 as a function of $t_{0}$ or the corresponding IFD $\omega_{D}\left(t_{0}\right)$. The measured spectral width of the aromatic $\mathrm{C}-\mathrm{H}$ vibration in polystyrene [Fig. 2(a)] decreases with increasing $\tau$, in agreement with the decreasing spectral width $\sigma_{D}$ of the vibrational excitation. Vice versa, the CARS from the gel [Fig. 2(b)], which is dominated by the spectrally nonresonant response of water, broadens in delay time and decreases in peak intensity with increasing pulse duration $\tau$. The measurements are in quantitative agreement with the calculated nonresonant CARS response for Gaussian pulses $\propto \tau_{G}^{-2} \exp \left(-4 t_{0}^{2} / 3 \tau_{G}^{2}\right)$, with a FWHM of $\tau \sqrt{3 / 2}$ and a peak scaling as $\tau^{-2}$. Conversely, the peak intensity of the resonant response keeps essentially constant as long as $\sigma_{D}$ is much larger than the probed Raman linewidth ${ }^{11} \quad \gamma=2 T_{2}^{-1}$ $\approx 27 \mathrm{~cm}^{-1}$. The ratio between resonant and nonresonant CARS accordingly increases with $\tau$ and saturates for $\tau \gg T_{2}$ $\approx 0.4 \mathrm{ps}$, as shown in the inset of Fig. 2(a) and also discussed in Ref. 3 .

To experimentally investigate the influence of the time ordering we recorded CARS spectra for different $\omega_{P}-\omega_{S}$ of $(3082,3026,2958) \mathrm{cm}^{-1}$. The corresponding data for 


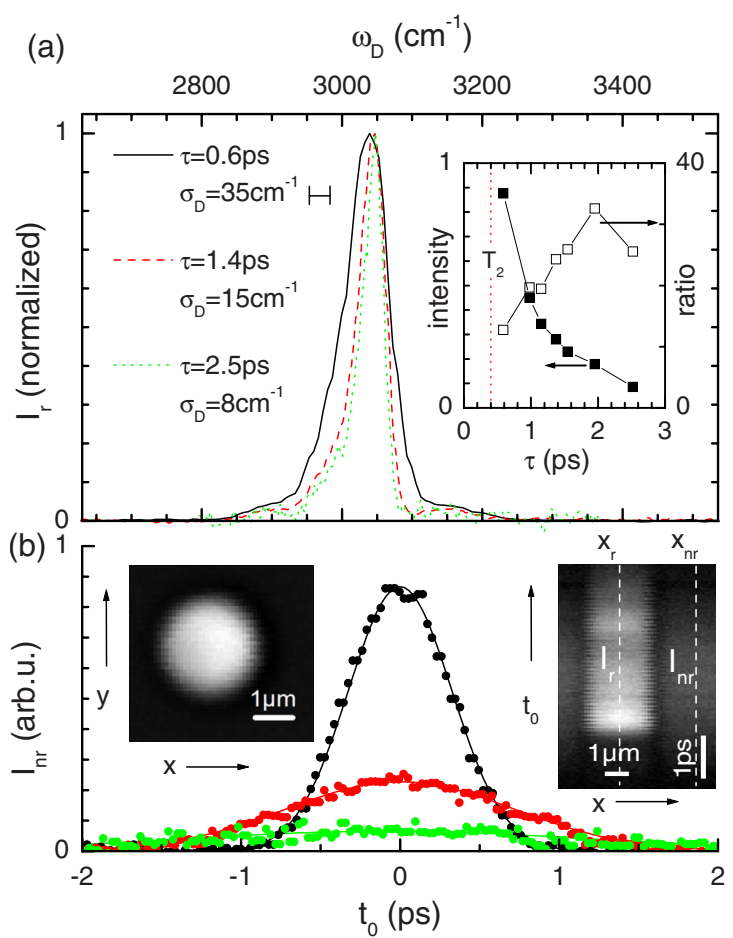

FIG. 2. (Color online) CARS spectra for $\omega_{P}-\omega_{S}=3082 \mathrm{~cm}^{-1}$ as a function of $t_{0}$ or $\omega_{D}\left(t_{0}\right)$ for different chirped pulse durations $\tau$ corresponding to different spectral resolutions as indicated. (a) Resonant CARS from a polystyrene bead (normalized to its maximum). (b) Nonresonant CARS in $2 \%$ agarose gel. Inset in (a): Resonant CARS peak intensity, (left) absolute and (right) relative to the nonresonant CARS intensity vs $\tau$. Insets in (b): (left) resonant CARS image of a $3 \mu \mathrm{m}$ polystyrene bead and (right) line scan vs delay $t_{0}$, from which CARS spectra inside and outside the bead are measured (dashed lines).

$\tau=1.4$ ps are given in Fig. 3(a). Additionally to the aromatic $\mathrm{C}-\mathrm{H}$, also the aliphatic vibrational bands at 2851 and $2901 \mathrm{~cm}^{-1}$ are observed. Clearly, $I_{\mathrm{r}}$ is larger for $\omega_{P}-\omega_{S}$ $>\omega_{\mathrm{r}}$. This is consistent with a maximum of $I_{\mathrm{r}}$ at $t_{0}=t_{0}^{\prime} \approx$ $-0.35 \tau_{G} /\left[1+\left(2 \tau_{G} / 3 T_{2}\right)^{4 / 3}\right]^{3 / 4}$, deduced from numerical simulations reported elsewhere, ${ }^{12}$ giving the modified resonance condition $\omega_{P}-\omega_{S}=\omega_{\mathrm{r}}-2 \beta t_{0}^{\prime} \approx 3070 \mathrm{~cm}^{-1}$. This modified condition is due to the effect of time ordering, giving the maximum $I_{\mathrm{r}}$ for resonant excitation at the optimum delay $\omega_{D}\left(t_{0}^{\prime}\right)=\omega_{\mathrm{r}}$.

In order to retrieve the intrinsic CARS spectrum ${ }^{13}$ we need to correct the measured CARS for the changing pulse overlap as given by the delay dependence of $I_{\mathrm{nr}}$. Spectra for different $\tau$ normalized accordingly are shown in Figs. 3(b)-3(d), and show the improvement of the spectral resolution with increasing $\tau$. Furthermore, we note that for $\tau$ $=0.6 \mathrm{ps}$ a spectral shift is observed, which is due to the interplay between excitation resonance and the time ordering. The influence of the time ordering on the spectral response function is negligible only for strongly chirped pulses $\left(\tau \gg T_{2}\right)$.

For a given pulse energy, we can deduce an optimum $\tau$ for imaging of a specific vibrational resonance. A good compromise between a high $I_{\mathrm{r}} / I_{\mathrm{nr}}$, improving the selectivity and increasing with $\tau$, and a high $I_{\mathrm{r}}$, improving the sensitivity and decreasing with $\tau$, is $\tau_{G}=T_{2}$. For this choice we have $t_{0}^{\prime}=$ $-0.23 T_{2}$ and $\omega_{P}-\omega_{S}=\omega_{\mathrm{r}}+0.55 / \tau_{0}$ (a shift by $20 \%$ of the pulse spectral FWHM), and we reach $34 \%$ of the maximum

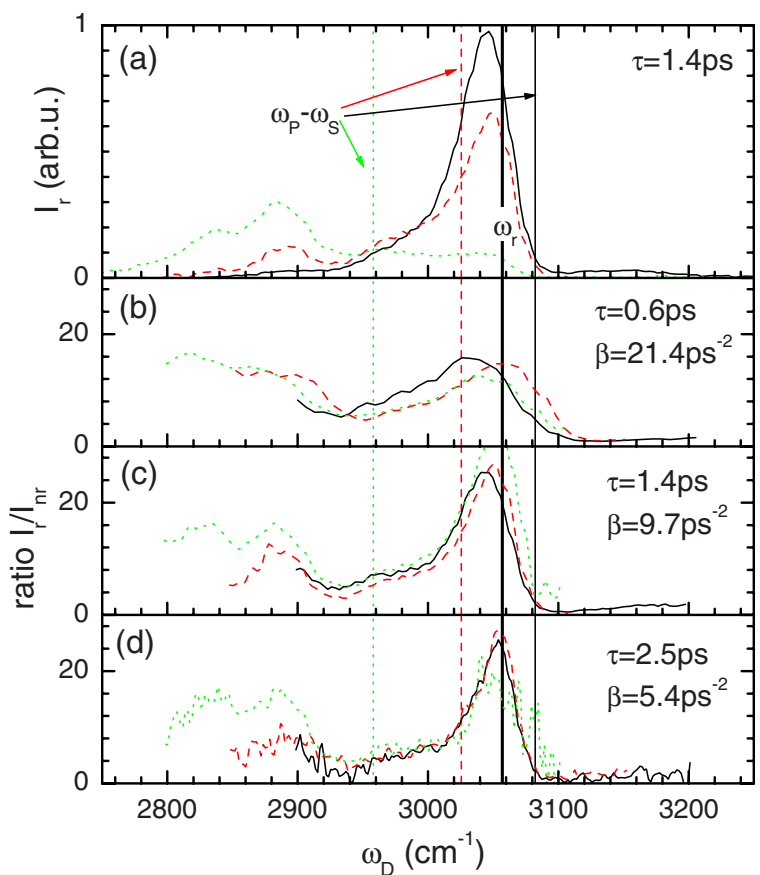

FIG. 3. (Color online) CARS in polystyrene vs $\omega_{D}\left(t_{0}\right)$ for different $\omega_{P}$ $-\omega_{S}$ as indicated by the vertical lines. (a) $I_{\mathrm{r}}$ for $\tau=1.4$ ps. [(b)-(d)] ratio $I_{\mathrm{r}} / I_{\mathrm{nr}}$ for $\tau$ as labeled.

$I_{\mathrm{r}} / I_{\mathrm{nr}}$ and $32 \%$ of the maximum $I_{\mathrm{r}}$. Note that in Ref. 7 it was $\tau / T_{2} \sim 5$, similar to our case for $\tau=2.5 \mathrm{ps}$.

In conclusion, we have demonstrated that using the second-order dispersion in glass results in a highly efficient, alignment insensitive, and cost-effective way to spectrally focus the entire bandwidth of $100 \mathrm{fs}$ pulses for singlefrequency CARS with high spectral resolution. Moreover, we showed that by controlling the difference frequency between pump and Stokes to optimize the temporal pulse overlap, the resonant CARS signal can be increased due to the impact of time ordering in the generation of CARS. Importantly, the proposed chirping method is also suited for usage with a single sub-10-fs source, resulting in a frequency-selective CARS system of fast and broadband tunability, yet compatible with femtosecond techniques such as SHG or TPF.

This work was funded by the UK Biotechnology and Biological Research Council (Grant No. BB/D001013/1).

${ }^{1}$ E. O. Potma and X. S. Xie, Opt. Photonics News, 40 (2004).

${ }^{2}$ J.-X. Cheng and X. Xie, J. Phys. Chem. B 108, 827 (2004).

${ }^{3}$ A. Volkmer, J. Phys. D 38, R59 (2005).

${ }^{4}$ M. Muller and A. Zumbusch, ChemPhysChem 8, 2156 (2007).

${ }^{5}$ J.-X. Cheng, A. Volkmer, L. Book, and X. Xie, J. Phys. Chem. B 106, 8493 (2002).

${ }^{6}$ K. P. Knutsen, B. Messer, R. Onorato, and R. Saykally, J. Phys. Chem. B 110, 5854 (2006).

${ }^{7}$ T. Hellerer, A. M. Enejder, and A. Zumbusch, Appl. Phys. Lett. 85, 25 (2004).

${ }^{8}$ N. Dudovich, D. Oron, and Y. Silberberg, Nature (London) 418, 512 (2002).

${ }^{9}$ J.-C. Diels and W. Rudolph, Ultrashort Laser Pulse Phenomena (Academic, New York, 1995).

${ }^{10}$ Zemax Glass catalog (http://www.zemax.com/).

${ }^{11}$ Polystyrene Raman spectrum (http://www.sigmaaldrich.com/).

${ }^{12}$ W. Langbein, I. Rocha-Mendoza, and P. Borri, J. Raman Spectrosc. (unpublished).

${ }^{13}$ The CARS spectra include nonresonant CARS from polystyrene, which is, however, negligible compared to the resonant CARS at $\omega_{\mathrm{r}}=3054 \mathrm{~cm}^{-1}$. 